\title{
Scale-Dependent Coherence of Terrestrial Vertebrate Biodiversity with Environment
}

Conor P. B. O'Malley ${ }^{1 \mathrm{a}}$, Gareth G. Roberts ${ }^{1 \mathrm{~b}}$, Philip D. Mannion ${ }^{2 \mathrm{c}}$, Jan Hackel ${ }^{3 \mathrm{~d}}$, Yanghua Wang ${ }^{1 \mathrm{e}}$ 8th February 2022

\footnotetext{
${ }^{1}$ Department of Earth Science \& Engineering, Imperial College London, Royal School of Mines, Prince Consort Road, London SW7 2BP, UK

${ }^{2}$ Department of Earth Sciences, University College London, London WC1E 6BT, UK

${ }^{3}$ Department of Trait Diversity and Function, Royal Botanic Gardens, Kew, London TW9 3AE, UK
}

${ }^{a}$ conor.omalley19@imperial.ac.uk

${ }^{b}$ gareth.roberts@imperial.ac.uk

${ }^{c}$ p.mannion@ucl.ac.uk

dJ.Hackel@kew.org

eyanghua.wang@imperial.ac.uk

Authorship Statement: C.O. and G.R. designed the project, developed code, interpreted results and wrote the manuscript with contributions from P.M., Y.W., J.H.; C.O performed the analyses and generated figures.

Data Accessibility Statement: If the manuscript is accepted, the data supporting the results will be archived on Zenodo: https://doi.org/10.5281/zenodo.XXXXXX.

Short Running Title: Coherence of Biodiversity with Environment

Keywords: biodiversity, topography, climate, spectral analysis

Article Type: Letter

Number of Words in Abstract/Main Text: 149/4013

Number of References: 53

Number of Figures/Tables/Reference Boxes: 3/0/0

Corresponding Author: Conor P. B. O'Malley, Royal School of Mines, Prince Consort Road, London SW7 2BP, UK. conor.omalley19@imperial.ac.uk 


\section{Abstract}

Disentangling contributions from environmental variables is crucial for explaining global biodiversity patterns. We use wavelet power spectra to separate wavelength-dependent trends across Earth's surface. Spectra reveal scale- and location-dependent coherence between species richness and topography $(E)$, annual precipitation $(P n)$, temperature $(T m)$ and temperature range $(\Delta T) .>97 \%$ of richness of carnivorans, bats, songbirds, hummingbirds and amphibians resides at wavelengths $\gtrsim 10^{3} \mathrm{~km} .30-69 \%$ is generated at scales $\gtrsim 10^{4} \mathrm{~km}$. At these scales, richness across the Americas is anti-correlated with $E$ and $\Delta T$, and positively correlated with $P n$ and $T m$. Carnivoran richness is incoherent with $\Delta T$, suggesting insensitivity to temperature seasonality. Conversely, amphibian richness is anti-correlated with $\Delta T$ at large scales. At scales $\lesssim 10^{3} \mathrm{~km}$, richness is highest within the tropics. Terrestrial plateaux exhibit coherence between carnivoran richness and $E$ at scales $\sim 10^{3} \mathrm{~km}$, reflecting contributions of orogeny/epeirogeny to biodiversity. Similar findings result from transects across other continents. Scale-dependent sensitivities of vertebrate populations to climate are revealed.

\section{Introduction}

Biological diversity is critical to many basic human needs, including health, food, water and shelter. It also plays an important role in moderating physical and chemical processes in natural environments (Balmford \& Bond, 2005; Barrett et al., 2011; Corenblit et al., 2011; Fei et al., 2014). Quantifying links between environment and biodiversity is crucial for understanding the response of ecosystems to climatic and physiographic change, and for conservation efforts (Araújo \& Rahbek, 2006; Hampe \& Petit, 2005; Norris et al., 2013). Many extrinsic processes postulated to control biodiversity (e.g. climate) are rapidly changing; therefore quantifying the strength of relationships between them is a pressing concern (Nogués-Bravo et al., 2018).

Environmental variables and species richness exhibit variance in space across a range of scales. However, it is unclear whether coherence between variables is uniform across all scales. As such, developing methodologies that can disentangle scale and location from biotic and environmental data to identify correlations is crucial. Here, we focus on quantifying coherence between species richness of continental vertebrate taxa and elevation, precipitation, temperature, and annual temperature range, which are postulated to drive biodiversity (e.g. Antonelli et al., 2018; Rahbek \& Graves, 2001). We do so by mapping coherence between biotic and environmental signals as a function of scale and location using wavelet spectral analyses. Unlike in spatial regression studies, these analyses inherently disentangle scale-dependent effects, and identify strength of correlation between variables at individual scales. 
Identifying links between biodiversity and environment has recently become significantly more tractable for three reasons. First, global patterns of species richness have been estimated with unprecedented detail, from horizontal scales as broad as continents, to those as fine as $\sim 10 \mathrm{~km}$ in wavelength (Jenkins \& Joppa, 2009; Jenkins et al., 2013, 2020). Second, values and variance of many environmental variables postulated to be responsible for determining distributions of species are now available globally at even higher resolution (e.g. Karger et al., 2017). Finally, wavelet spectral methods, which can identify the locations and scales at which signals (e.g. spatial series of taxa) are generated, as well as coherence and phase differences (offsets) between series such as species richness, topography and climate, are now established (see Materials and Methods; Grinsted et al., 2004; Torrence \& Compo, 1998). These kinds of analyses are key to understanding how the changing global climate will affect the distribution of biodiversity across Earth.

\section{Materials and Methods}

\subsection{Species Richness Data}

Figure 1a-f shows species richness per $10 \times 10 \mathrm{~km}$ cell for all mammals (Mammalia), carnivorans (Carnivora), bats (Chiroptera), songbirds (Passeriformes), hummingbirds (Trochilidae), and amphibians (Amphibia). These data reinforce well-known large-scale observations, e.g. the latitudinal diversity gradient (LDG), but also contain evidence of significant complexity across scales of interest, here wavelengths between 10-10 $\mathrm{km}$ (Hillebrand, 2004; Willig et al., 2003). We examine species richness trends in this study, since it is the easiest biodiversity metric to calculate, having been done so for a wide range of taxa. Here, we focus on terrestrial taxa since terrestrial surface environmental conditions are best-mapped, as is terrestrial vertebrate biodiversity. Similar analysis is possible for marine taxa, invertebrates, plants etc., and for metrics other than species richness, for example range sizes and trophic interactions.

Species richness is here defined as number of species of a given taxon within a $10 \times 10 \mathrm{~km}$ square. We use the grids compiled by Jenkins et al. (2013), which were generated by combining maps of species distributions, and counting the number of overlapping polygons in a given cell. For birds, the species richness data were calculated from breeding ranges compiled by BirdLife International NatureServe (2011). For amphibians and mammals, the data were based on range maps generated by the International Union for Conservation of Nature (2021). A minimum grid spacing of $10 \mathrm{~km}$ yields a minimum scale for wavelet spectral analysis of $\sim 20 \mathrm{~km}$ (see Materials and Methods; Torrence \& 
Compo, 1998). Species richness varies as a function of the spatial range characteristics of a study, particularly "grain", i.e. piece-wise horizontal resolution within a study (Gaston, 2000; Palmer \& White, 1994; Willig et al., 2003). By using a constant grain (i.e. "focus" or grid spacing) of $10 \mathrm{~km}$, challenges associated with comparing results generated using different grains are avoided (Willig et al., 2003). Here, scale-dependent trends are calculated as a function of "extent" rather than "grain" sensu Palmer \& White (1994). Latitudinal terrestrial averages of species richness and environmental data, and their wavelet transforms are shown in Supporting Information Figures S25-S29.

Latitudinal transects through terrestrial vertebrate richness data are shown in Figure 2. We focus on the Americas, where transects can be generated that encompass almost all of Earth's latitudinal range (Figures $1 \& 2$ : $\left.A-\mathrm{A}^{\prime}\right)$. Transects through data for Australia $\left(\mathrm{B}-\mathrm{B}^{\prime}\right)$, Africa $\left(\mathrm{C}-\mathrm{C}^{\prime}\right)$, Eurasia $\left(\mathrm{D}-\mathrm{D}^{\prime}\right)$ and global averages are shown in Supporting Information (Figures S7-S29). We have examined how uncertainties in species richness could contribute to uncertainties in calculated spectra and coherence by adding uniformly distributed (white) noise to transects before they are transformed into the spectral domain (Supporting Information Figure S5).

\subsection{Environmental Variable Data}

Figures $1 \mathrm{~g}-\mathrm{i}$ and $2 \mathrm{~m}, \mathrm{o}, \mathrm{q}$ and $\mathrm{s}$ show examples of maps and cross sections through elevation and climatic data which we use, from the ETOPO1 and CHELSA datasets, respectively (Amante \& Eakins, 2009; Karger et al., 2017).

The global elevation grid ETOPO1 has a horizontal resolution of 1 arc-minute (Figure 1g; Amante \& Eakins, 2009). It is primarily generated from $\sim 30 \mathrm{~m}$ resolution Shuttle Radar Topography Mission (SRTM30) data and includes interpolated coastlines and satellite altimetry (Jarvis et al., 2008). Amante \& Eakins (2009) suggest a mean vertical error of $\sim 10$ metres for ETOPO1. Since the horizontal resolution of this dataset is approximately $1.8 \mathrm{~km}$, wavelet transformation of topography in this case would have a minimum scale of $\sim 3.6 \mathrm{~km}$ (see below; Torrence \& Compo, 1998). We downsampled the data to a horizontal resolution of $10 \mathrm{~km}$ using Generic Mapping Tools to match resolution of species richness grids (Wessel et al., 2019).

Annual mean values for climatic data, from 1981-2010, were extracted from the Climatologies at High Resolution for the Earth's Land Surface Areas (CHELSA) dataset (Karger et al., 2017). CHELSA was generated by applying corrections to the ERA-Interim climatic reanalysis and has a horizontal resolution of up to 30 arc-seconds (Dee et al., 2011). Temperature data were corrected for elevation 
above sea level and precipitation rates were corrected using wind direction, valley exposition and boundary layers. Precipitation rate is weakly dependent on elevation. These values were successfully benchmarked against alternative climatology data and models: WorldClim, TRMM , GPCC and GHCN (Hijmans et al., 2005; Goddard Earth Sciences Data and Information Services Center, 2017; Lawrimore et al., 2011; Schneider et al., 2014). The data were down-sampled to $10 \mathrm{~km}$ prior to spectral analyses.

\subsection{Continuous Wavelet Transform}

Spatial series, $x_{n}$, of species richness or environmental variables were transformed into distancewavenumber space using continuous wavelet transforms (for practical guide, see Torrence \& Compo, 1998). The transform convolves uniformly sampled spatial series with a mother wavelet, $\psi$. The Morlet wavelet with dimensionless frequency $\omega_{\circ}=6$ is used in this study, although other mother wavelets are investigated in Supporting Information Figure S6. Use of different mother wavelets (Morlet, order $\omega_{\circ}=4,8$; Paul, order $m=2,4,6$; derivative of Gaussian, order $m=2,4,6$ ) does not significantly change patterns of mapped power, and distance-averaged power shows similar trends to the results presented here. The mother wavelet is scaled and translated along spatial series to reveal variations in amplitude as a function of scale, $s$, and position, $x_{n}$. Sampling interval $\delta j=10 \mathrm{~km}, n=0,1 \ldots N-1$, where $N$ is number of measurements. The wavelet transformation is

$$
W_{n}(s)=\sum_{n^{\prime}=0}^{N-1} x_{n} \psi^{*}\left[\frac{\left(n^{\prime}-n\right) \delta t}{s}\right],
$$

where $*$ denotes the complex conjugate. We use the mlpy Python module to transform the spatial series (Albanese et al., 2012), which is based on the methods summarized by Torrence \& Compo (1998). Spatial series were mirrored across the $x$ (distance) and $y$ (dependent variable) axes to reduce edge effects (Roberts et al., 2019). Inverse transforms were generated for each signal to quantify fidelity of transformed series. Median difference between input signals and inverse transforms were always $\leq 0.9 \%$. The distance-averaged power spectrum, which yields similar results to Fourier transformation, is given by

$$
\phi(s)=\frac{1}{N} \sum_{x=0}^{N}\left|W_{n}(s)\right|^{2} .
$$

In Figure 3, we plot rectified distance-averaged power $\phi_{r}=\phi(s) s^{-1}$ after Liu et al. (2007). We calculate distance-averaged power within and outside of the tropics, but note that in those calculations, power was normalized by the proportion of the transect within/outside of the tropics respectively. Therefore there is no bias in distance-averaged power if the transect has a greater distance within/outside of tropical latitudes. Best-fitting spectral slopes were identified using simple one- and two-slope models after Roberts et al. (2019) (see Supporting Information). 


\subsection{Cross Wavelet Power \& Wavelet Coherence}

Cross wavelet power is calculated to identify signals in separate spatial series (e.g. amphibian richness and precipitation) that have large amplitudes located at the same position in distance-wavenumber space. To facilitate comparison, signals are normalized to zero mean and unit variance prior to transformation. The normalized signals $X$ and $Y$, are transformed to yield $W^{X}$ and $W^{Y}$. Cross wavelet power $W^{X Y}$ is calculated such that

$$
W^{X Y}=W^{X} W^{Y^{*}}
$$

Wavelet coherence, $R_{n}^{2}$, is calculated to identify parts of signals that are coherent, but not necessarily of common high amplitude, such that

$$
R_{n}^{2}(s)=\frac{\left|S\left\{s^{-1} W_{n}^{X Y}(s)\right\}\right|^{2}}{S\left\{s^{-1}\left|W_{n}^{X}(s)\right|^{2}\right\} \cdot S\left\{s^{-1}\left|W_{n}^{Y}(s)\right|^{2}\right\}},
$$

where $s, n$ and $W_{n}(s)$ are as in Equation 1. $S$ is an operator that smooths along distance and scale (Grinsted et al., 2004).

Since each of the studied signals broadly exhibits a red noise relationship (autocorrelation) between power and wavenumber, there is a chance that sections of transects of different variables could correlate by chance, without true interdependence. Therefore, it is important to calculate the coherence between each pair of signals, and not simply the cross wavelet power. The $90 \%$ significance limit for coherence, which was used to mask Figure 3, depends only on scale and not position, and was calculated using Monte Carlo methods with the PyCWT Python module (Grinsted et al., 2004; Krieger et al., 2020). The minimum bound for coherence per scale, for each transect, was calculated from cross wavelet power spectral analysis of 300 random signals, which follow a red noise spectral relationship, generated by the same autocorrelation coefficient as the input signals, and having the same length $N$ as the input signals.

The local phase difference (angular offset, $0 \leq a \leq 2 \pi$ ) of two signals is given by the complex argument of their cross wavelet transform, $\arg \left(W^{X Y}\right)$ (Grinsted et al., 2004). Figure 3 indicates phase difference as arrows measured from horizontal: in-phase, $a=0, \triangleright$; anti-phase, $a=\pi, \triangleleft$. A working example for species richness and elevation, including continuous wavelet transformation, cross wavelet power and wavelet coherence calculations, can be found at https://doi.org/10.5281/ zenodo.XXXXXX. 


\section{Results}

Spectral analyses of American vertebrate species richness and environmental variables are shown in Figures 2 and 3. Figure 2 shows that highest spectral power, $\phi\left(\propto z^{2}\right.$, where $z$ is signal amplitude), is concentrated at largest scales for all taxa and environmental variables studied. Dependent on taxonomic group, from $96 \%$ to almost $100 \%$ of power resides at wavelengths $>10^{3} \mathrm{~km}$. $29-74 \%$ of power resides at wavelengths $\gtrsim 10^{4} \mathrm{~km}$. These results reinforce the notion that species richness is dominated by long wavelength, latitudinal, variability. A guide to scale-dependence and self-similarity of spatial series is the color of spectral noise that they possess. For example, red (Brownian) noise occurs when $\phi \propto k^{-2}$, where $k$ is wavenumber or spatial frequency, proportional to $1 /$ wavelength, indicating selfsimilarity. Pink noise occurs when $\phi \propto k^{-1}$, and white noise indicates that power is equal across all scales, $\phi \propto 1$.

Species richness tends to have a pink noise spectrum (see Supporting Information for slope fitting). Thereby, shorter wavelength features in species richness signals tend to have the lowest amplitudes and comprise relatively little (few \%) of species richness signal at a particular location. Mammals and bats are better characterized by red noise at long wavelengths. This result implies self-similarity across scales, and that signal amplitudes decrease even more rapidly with decreasing wavelength than for other taxa. At wavelengths $\gtrsim 10^{3} \mathrm{~km}$, species richness power for amphibians is best characterized as blue noise, i.e. $\phi \propto k^{1}$. This trend is not observed along the entire transect, but indicates that short wavelength features can be increasingly important contributors to amphibian richness (see Figure 2f). A single spectral slope akin to pink noise can adequately fit the amphibian richness spectrum (see Supporting Information Figure S1f, p).

To assess the impact of uncertainties for these results, white noise was added to the amphibian transect in a systematic set of tests. These tests examined changes in calculated spectra when noise with maximum amplitudes of $10 \%, 50 \%$ and $100 \%$ of the standard deviation of the original signal's amplitude (in this case $=24$ species per pixel) was added to the transect prior to transformation. These tests included adding noise at wavelengths $\lesssim 100, \lesssim 1000$ and $\lesssim 10,000 \mathrm{~km}$ (Supporting Information Figure S5). As expected, these tests indicated that spectral power is least likely to be well constrained at short wavelengths. Nonetheless, these tests indicate that even high amplitude uniformly distributed noise does not significantly change the overall spectral characteristics of terrestrial species richness.

Although almost no power is concentrated below wavelengths of $\sim 100 \mathrm{~km}$ for any of the taxa examined here, there are some parts of some wavelet transforms which show increased power in the range $\sim 300-1000 \mathrm{~km}$. This deviation, away from a broadly monotonic decrease in power towards 
shorter wavelengths, is driven principally by species richness within tropical latitudes, and is especially prominent for songbirds, hummingbirds and amphibians (Figure $2 \mathrm{~h}, \mathrm{j}, \mathrm{l}$ ). Supporting Information Figure S3a-f shows that at wavelengths $\gtrsim 1000 \mathrm{~km}$, there is no notable difference between power in species richness within or outside the tropics. However, at wavelengths $\lesssim 1000 \mathrm{~km}$, there is significantly greater power for regions within the tropics. This trend arises since power spectral slopes remain close to -2 at shorter wavelengths outside of the tropics (i.e. red noise; Supporting Information Figure S3), before increasing to be closer to -1 (i.e. pink noise). We suggest that these results are consistent with the concept that topography in tropical regions can generate higher species richness towards the equator via the increased effectiveness of relief at isolating species (Janzen, 1967). We find the effect has a greatest impact on species richness power of hummingbirds and amphibians; the impact on bats and songbirds richness appears to be more modest. Tropical increases in species richness of carnivorans, and mammals more generally, are much more subdued (Supporting Information Figure S3).

Elevation transects exhibit red and pink noise spectral characteristics at wavelengths $\gtrsim 10^{3} \mathrm{~km}$ and $\lesssim 10^{3} \mathrm{~km}$, respectively, which we note is similar to distance-averaged power from wavelet transforms of longitudinal river profiles and other topographic transects (Supporting Information Figures S1g, S1q, S2g, S2q; Roberts et al., 2019; Wapenhans et al., 2021). Precipitation rate, temperature and annual temperature range can also be characterized as red and pink noise (Figures $\mathrm{S} 1 \mathrm{~h}-\mathrm{j}, \mathrm{r}-\mathrm{t} \& \mathrm{~S} 2 \mathrm{~h}-\mathrm{j}$, $\mathrm{r}-\mathrm{t}$ ). Similar results are obtained for transects through Africa, Eurasia and Australia, as well as across global, latitudinally-averaged sections (see Supporting Information).

\subsection{Coherence between Taxa and Environment}

Visual inspection of Figure 2 indicates that there is strong, location- and scale-dependent, similarity between the wavelet transforms of transects through species richness and environmental variables. To quantify the strength of these relationships we calculate cross wavelet power, which identifies co-located high amplitudes in the location-scale domain, and wavelet coherence (see Materials and Methods). In the main manuscript, we show results from carnivorans (which are similar to those for mammals generally), and amphibians (which are similar to those for bats, songbirds and hummingbirds). See Supporting Information Figure S3 for analysis of those other taxa.

Figure 3a shows cross wavelet power between species richness of carnivorans along transect $\mathrm{A}-\mathrm{A}^{\prime}$ and elevation. Almost no short-wavelength $\left(<10^{3} \mathrm{~km}\right)$ features are coherent above a $90 \%$ confidence limit (see Materials and Methods). These short wavelength regions contain almost no cross wavelet power; $94 \%$ of all cross power is in the region of high coherence colored on Figure 3a, which accounts for $30 \%$ of the location-scale domain. $79 \%$ of the area of the cross wavelet spectrum that is signific- 
antly coherent resides at wavelengths $\gtrsim 10^{3} \mathrm{~km}$. Distance-averaged cross wavelet power for all parts of the power spectrum, not just those parts which are coherent above the $90 \%$ significance threshold, is shown to the right of each panel, on a logarithmic scale. Full, unmasked, plots of cross wavelet power are shown in Supporting Information Figure S4. Distance-averaged cross wavelet power between all taxa and environmental variables studied is shown in Figure S3g-ad.

Cross wavelet power between amphibians and elevation is also highest at long wavelengths, although overall there is a smaller proportion of the two signals that is coherent: $78 \%$ of the plot region is masked in Figure 3e. Only a small part of the cross wavelet transform for amphibians and elevation is coherent below wavelengths of $\sim 5000 \mathrm{~km}$, and that part lies near the centre of the transect, i.e. within the tropics. Distance-averaged power outside the tropics, plotted to the right of Figure 3e, is an order of magnitude lower than within the tropics, especially at shorter wavelengths. This observation is in contrast to cross power between species richness of carnivorans and elevation, where there is almost no difference within the tropics and outside the tropics, across all scales. These results may indicate that carnivorans are less affected by "mountain passes" (sensu Janzen, 1967) in the tropics, compared with amphibians (cf. Antonelli et al., 2018; Eronen et al., 2015; Rahbek et al., 2019; Rolland et al., 2015). Carnivoran species richness is most coherent with elevation and mean annual temperature at wavelengths $\sim 10^{3} \mathrm{~km}$ atop terrestrial plateaux (e.g. Rocky-Mountains-Colorado Plateau and Altiplano, between $4000-7000 \mathrm{~km}$ and 13,000 - 14,000 km distance along transect $\mathrm{A}-\mathrm{A}^{\prime}$, respectively; Figures 1-3). An obvious interpretation is the local importance of tectonics for determining biodiversity (Antonelli et al., 2018).

Coherent cross wavelet power between species richness of carnivorans and amphibians, mean annual precipitation rate, temperature and annual temperature range is shown in Figure $3 \mathrm{~b}-\mathrm{d}, \mathrm{f}-\mathrm{h}$. Cross power between amphibian species richness and precipitation rate, temperature, and temperature range is high within the tropics, whereas those differences are absent or reduced for carnivorans. Furthermore, for these three climatic variables, there is much weaker coherence with carnivoran species richness. Carnivorans appear less sensitive to changes in those variables compared with amphibians. Calculated phase indicates long-wavelength anticorrelation between elevation and species richness for both carnivorans and amphibians (left-pointing arrows in Figure 3a and e; phase angle, $a=\pi$; see Materials and Methods). Highly coherent long-wavelength anticorrelation between amphibian species richness and annual temperature range is also observed across the entire transect. Highly coherent, long-wavelength cross power between precipitation rate or temperature and species richness of both carnivorans and amphibians is in phase, i.e. there is positive correlation at these scales. This result is in agreement with the idea that faster diversification rates drive species richness, since it suggests that both taxa benefit from increased energy and high productivity associated with greater availability of 
heat and water (cf. Allen et al., 2006).

\subsection{Global and Local Species Richness and Environment}

These American results can be compared to transects from Australia, Eurasia and Africa. For Australia, similar trends in power spectral slopes, distance-averaged power and cross wavelet power are observed (Figure 1: B-B'; Supporting Information Figures S8-S10). However, there is almost no difference in power or cross power between tropical regions and regions outside the tropics. We note, however, that the transect does not include the entirety of the tropics. Signals are mostly coherent at wavelengths $\gtrsim 10^{3} \mathrm{~km}$, and the same pattern of correlation/anticorrelation is observed with climatic variables (Supporting Information Figure S11). In Africa, songbirds and amphibians have greater species richness power within the tropics but the differences are not as stark as for the Americas (Figure 1: $\mathrm{C}-\mathrm{C}^{\prime}$; Supporting Information Figure S16a-f). This result may reflect differences in Cenozoic paleoclimatic history between Africa and the Americas (Hagen et al., 2021). The greatest difference between cross power within the tropics and outside the tropics is for precipitation rate, suggesting that water availability controls species richness for all African taxa studied here. Wavelet coherence indicates that, across Africa, carnivoran species richness does not correlate with environmental variables, whereas species richness of amphibians is strongly positively correlated with precipitation rate at long wavelengths (Buckley \& Jetz, 2007). Anticorrelation is observed between amphibian species richness and temperature across Africa. Results for Eurasia are dominated by the presence of the Tibetan Plateau, and the low proportion of the transect within tropical latitudes (Figure 1: D- $\mathrm{D}^{\prime}$; Supporting Information Figures S19-S24). Similar trends to the Americas are observed, albeit with generally lower cross power and coherence.

Mean terrestrial values of each variable across all latitudes globally were transformed into the location-scale domain. Distance-averaged wavelet power spectra of the resulting transects have spectral slopes between -2 and -1 (red to pink noise), reflecting the importance of long-wavelength trends. Species richness power for all taxa except Mammalia and Carnivora is at least an order of magnitude lower outside of tropical latitudes, at wavelengths $\lesssim 3000 \mathrm{~km}$, consistent with results obtained from transforming the American transect (Figures 2 and 3). This result suggests that the increase in species richness power at short wavelengths may be a global phenomenon reflecting sensitivity of tropical species to local climatic effects.

\section{Discussion}

A principal result of this study is that terrestrial species richness tends to be most coherent with topography, precipitation and temperature at long wavelengths $\left(>10^{3} \mathrm{~km}\right)$. These results indicate that 
large-scale variation in tectonic and climatic processes play a governing role in generating the LDG (Field et al., 2009). However, our results also indicate that the distribution of taxa, and their coherence and phase with environmental variables, is highly location- and scale-dependent. For example, whereas carnivorans and amphibians are in phase and coherent with mean annual precipitation and temperature at wavelengths $>10^{4} \mathrm{~km}$, that is not true at smaller scales (i.e. shorter wavelengths). Significant deviations from the LDG indicate that external variables such as elevation, climatic patterns and tectonic history, play important roles in determining biodiversity at specific locations and scales (e.g. Archibald et al., 2010, 2013; Hagen et al., 2021; Mannion et al., 2014; Saupe, 2021; Song et al., 2020).

Spectral analyses highlight the importance of the tropics for biodiversity, in particular for amphibians where local changes in elevation and mean annual temperature (but not annual temperature range) are highly coherent with species richness. These results are consistent with the idea that increased resource availability at the tropics may generate higher primary productivity, supporting a greater number of individuals within a given area (i.e. higher carrying capacity), and therefore a greater number of different species (e.g. Fritz et al., 2016; Gillman et al., 2015; Hawkins et al., 2003; Kessler et al., 2014). Our results support the suggestion that elevated topography at the tropics is more likely to result in increased species diversity when compared to higher latitudes (Janzen, 1967; Polato et al., 2018). However, this trend is not uniformly observed across taxa and for all continents. Species richness of carnivorans, for example, has no significant coherence with elevation or temperature range in the tropics, which suggests that this group is largely unaffected by the challenges posed by tropical mountain ranges. Power spectral slopes for such taxa are steeper (more negative) at shorter wavelengths, whereas more environmentally-sensitive taxa, such as hummingbirds and amphibians, have shallower spectral slopes at longer wavelengths within tropical latitudes.

Cross wavelet power and coherence indicate that species richness is decoupled from short wavelength $\left(\lesssim 10^{3} \mathrm{~km}\right.$ ) changes in elevation, temperature, annual temperature range and precipitation at nearly all locations, except for certain taxa within the tropics. Locally, uplifted topography can be highly coherent with species richness. Trends across the Americas are reflected in global, latitudinally-averaged, transects and for other continents. In general, the species richness of taxa such as hummingbirds and amphibians is strongly and positively correlated with precipitation rate and temperature, except in Africa, where high temperatures may limit availability of water. Crucially, these results could be used to predict the changes in biodiversity that could arise from different future Earth climate change scenarios.

In summary, wavelet power spectral analysis provides insight into the coherence between species 
richness and environmental variables. Species richness is shown to vary as a function of location and scale. Comparisons with topography, temperature and precipitation show that species richness tends to be highly coherent with external forcing at large scales (wavelengths $>10^{4} \mathrm{~km}$ ). Phase difference between signals reveals that species richness is in-phase with precipitation and temperature, and antiphase with elevation and annual temperature range, at these scales. However, these relationships are dependent on scale and taxon. At smaller scales, richness of bats, songbirds, hummingbirds and amphibians tends to be greatest in the tropics, where calculated coherence highlights the importance of topography and temperature range for determining species richness. Carnivorans, in contrast, show little coherence with environmental variables at these scales in the tropics. They are instead most coherent in the vicinity of the Colorado Plateau and Altiplano. These observations suggest that large scale $\left(>10^{3} \mathrm{~km}\right)$ variations in environmental variables determine almost all of the distribution of terrestrial vertebrates. Smaller scale $\left(\lesssim 10^{3} \mathrm{~km}\right)$ variation can play an important role locally, particularly within the tropics. These results highlight the importance of environment change at the scale of tens degrees of latitude, and local changes in tropical environment, for determining biodiversity.

\section{Acknowledgements}

C.O. was supported by the Leverhulme Trust (Grant: RPG-2019-073). P.D.M. was supported by a Royal Society University Research Fellowship (UF160216). Figures were generated using Generic Mapping Tools v6.2.0 (Wessel et al., 2019). We thank P. Ball, C. Donaldson, F. Richards and A. Whittaker for helpful discussion.

\section{References}

Albanese, D., Visintainer, R., Merler, S., Riccadonna, S., Jurman, G., \& Furlanello, C., 2012. mlpy: Machine Learning Python.

Allen, A. P., Gillooly, J. F., Savage, V. M., \& Brown, J. H., 2006. Kinetic effects of temperature on rates of genetic divergence and speciation, PNAS, 103(24), 9130-9135.

Amante, C. \& Eakins, B. W., 2009. ETOPO1 Arc-minute global relief model: Procedures, data sources and analysis, Tech. rep., NOAA, Boulder, Colorado.

Antonelli, A., Kissling, W. D., Flantua, S. G. A., Bermúdez, M. A., Mulch, A., Muellner-Riehl, A. N., Kreft, H., Linder, H. P., Badgley, C., Fjeldså, J., Fritz, S. A., Rahbek, C., Herman, F., Hooghiemstra, H., \& Hoorn, C., 2018. Geological and climatic influences on mountain biodiversity, Nature Geoscience, 11, 718-725. 
Araújo, M. B. \& Rahbek, C., 2006. How Does Climate Change Affect Biodiversity?, Science, 313, $1396-1397$.

Archibald, S. B., Bossert, W. H., Greenwood, D. R., \& Farrell, B. D., 2010. Seasonality, the latitudinal gradient of diversity, and Eocene insects, Paleobiology, 36(3), 374-398.

Archibald, S. B., Greenwood, D. R., \& Mathewes, R. W., 2013. Seasonality, montane beta diversity, and Eocene insects: Testing Janzen's dispersal hypothesis in an equable world, Palaeogeography, Palaeoclimatology, Palaeoecology, 371, 1-8.

Balmford, A. \& Bond, W., 2005. Trends in the state of nature and their implications for human well-being, Ecology Letters, 8(11), 1218-1234.

Barrett, C. B., Travis, A. J., \& Dasgupta, P., 2011. On biodiversity conservation and poverty traps, PNAS, 108(34), 13907-13912.

BirdLife International NatureServe, 2011. Bird Species Distribution Maps of the World, BirdLife International, Cambridge, UK.

Buckley, L. B. \& Jetz, W., 2007. Environmental and historical constraints on global patterns of amphibian richness, Proceedings of the Royal Society B, 274, 1167-1173.

Corenblit, D., Baas, A. C., Bornette, G., Darrozes, J., Delmotte, S., Francis, R. A., Gurnell, A. M., Julien, F., Naiman, R. J., \& Steiger, J., 2011. Feedbacks between geomorphology and biota controlling Earth surface processes and landforms: A review of foundation concepts and current understandings, Earth-Science Reviews, 106(3-4), 307-331.

Dee, D. P., Uppala, S. M., Simmons, A. J., Berrisford, P., Poli, P., Kobayashi, S., Andrae, U., Balmaseda, M. A., Balsamo, G., Bauer, P., Bechtold, P., Beljaars, A. C., van de Berg, L., Bidlot, J., Bormann, N., Delsol, C., Dragani, R., Fuentes, M., Geer, A. J., Haimberger, L., Healy, S. B., Hersbach, H., Hólm, E. V., Isaksen, L., Kållberg, P., Köhler, M., Matricardi, M., Mcnally, A. P., Monge-Sanz, B. M., Morcrette, J. J., Park, B. K., Peubey, C., de Rosnay, P., Tavolato, C., Thépaut, J. N., \& Vitart, F., 2011. The ERA-Interim reanalysis: Configuration and performance of the data assimilation system, Quarterly Journal of the Royal Meteorological Society, 137(656), 553-597.

Eronen, J. T., Janis, C. M., Chamberlain, C. P., \& Mulch, A., 2015. Mountain uplift explains differences in Palaeogene patterns of mammalian evolution and extinction between North America and Europe, Proceedings of the Royal Society B: Biological Sciences, 282(20150136), 1-8.

Fei, S., Phillips, J., \& Shouse, M., 2014. Biogeomorphic impacts of invasive species, Annual Review of Ecology, Evolution, and Systematics, 45, 69-87. 
Field, R., Hawkins, B. A., Cornell, H. V., Currie, D. J., Diniz-Filho, J. A. F., Guégan, J.-F., Kaufman, D. M., Kerr, J. T., Mittelbach, G. G., Oberdorff, T., O'Brien, E. M., \& Turner, J. R. G., 2009. Spatial species-richness gradients across scales: A meta-analysis, Journal of Biogeography, 36(1), $132-147$.

Fritz, S. A., Eronen, J. T., Schnitzler, J., Hof, C., Janis, C. M., Mulch, A., Böhning-Gaese, K., \& Graham, C. H., 2016. Twenty-million-year relationship between mammalian diversity and primary productivity, PNAS, 113(39), 10908-10913.

Gaston, K. J., 2000. Global patterns in biodiversity, Nature, 405(6783), 220-227.

Gillman, L. N., Wright, S. D., Cusens, J., McBride, P. D., Malhi, Y., \& Whittaker, R. J., 2015. Latitude, productivity and species richness, Global Ecology and Biogeography, 24(1), 107-117.

Goddard Earth Sciences Data and Information Services Center, 2017. TRMM (TMPA/3B43) Rainfall Estimate L3 1 Month 0.25 Degree x 0.25 Degree V7.

Grinsted, A., Moore, J. C., \& Jevrejeva, S., 2004. Application of the cross wavelet transform and wavelet coherence to geophysical time series, Nonlinear Processes in Geophysics, 11(5/6), 561-566.

Hagen, O., Skeels, A., Onstein, R. E., Jetz, W., \& Pellissier, L., 2021. Earth history events shaped the evolution of biodiversity across tropical rainforests, PNAS, 118(40), 1-11.

Hampe, A. \& Petit, R. J., 2005. Conserving biodiversity under climate change: The rear edge matters, Ecology Letters, 8(5), 461-467.

Hawkins, B. A., Porter, E. E., \& Diniz-Filho, J. A. F., 2003. Productivity and history as predictors of the latitudinal diversity gradient of terrestrial birds, Ecology, 84(6), 1608-1623.

Hijmans, R. J., Cameron, S. E., Parra, J. L., Jones, P. G., \& Jarvis, A., 2005. Very high resolution interpolated climate surfaces for global land areas, International Journal of Climatology, 25(15), $1965-1978$.

Hillebrand, H., 2004. On the generality of the latitudinal diversity gradient, American Naturalist, 163(2), 192-211.

International Union for Conservation of Nature, 2021. The IUCN Red List of Threatened Species.

Janzen, D. H., 1967. Why Mountain Passes are Higher in the Tropics, The American Naturalist, 101(919), 233-249.

Jarvis, A., Reuter, H. I., Nelson, A., \& Guevara, E., 2008. Hole-filled seamless SRTM data V4. 
Jenkins, C. N. \& Joppa, L., 2009. Expansion of the global terrestrial protected area system, Biological Conservation, 142(10), 2166-2174.

Jenkins, C. N., Pimm, S. L., \& Joppa, L. N., 2013. Global patterns of terrestrial vertebrate diversity and conservation, PNAS, 110(28), E2603-E2610.

Jenkins, J., Stephenson, S. N., Martínez-Garzón, P., Bohnhoff, M., \& Nurlu, M., 2020. Crustal Thickness Variation Across the Sea of Marmara Region, NW Turkey: A Reflection of Modern and Ancient Tectonic Processes, Tectonics, 39(7), 1-18.

Karger, D. N., Conrad, O., Böhner, J., Kawohl, T., Kreft, H., Soria-Auza, R. W., Zimmermann, N. E., Linder, H. P., \& Kessler, M., 2017. Climatologies at high resolution for the earth's land surface areas, Scientific Data, 4(170122), 1-20.

Kessler, M., Salazar, L., Homeier, J., \& Kluge, J., 2014. Species richness-productivity relationships of tropical terrestrial ferns at regional and local scales, Journal of Ecology, 102(6), 1623-1633.

Krieger, S., Freij, N., Brazhe, A., Torrence, C., \& Compo, G. P., 2020. PyCWT: A Python module for continuous wavelet spectral analysis.

Lawrimore, J. H., Menne, M. J., Gleason, B. E., Williams, C. N., Wuertz, D. B., Vose, R. S., \& Rennie, J., 2011. An overview of the Global Historical Climatology Network monthly mean temperature data set, version 3, Journal of Geophysical Research Atmospheres, 116(19), 1-18.

Liu, Y., Liang, X. S., \& Weisberg, R. H., 2007. Rectification of the bias in the wavelet power spectrum, Journal of Atmospheric and Oceanic Technology, 24(12), 2093-2102.

Mannion, P. D., Upchurch, P., Benson, R. B. J., \& Goswami, A., 2014. The latitudinal biodiversity gradient through deep time, Trends in Ecology and Evolution, 29(1), 42-50.

Nogués-Bravo, D., Rodríguez-Sánchez, F., Orsini, L., de Boer, E., Jansson, R., Morlon, H., Fordham, D. A., \& Jackson, S. T., 2018. Cracking the Code of Biodiversity Responses to Past Climate Change, Trends in Ecology and Evolution, 33(10), 765-776.

Norris, R. D., Kirtland Turner, S., Hull, P. M., \& Ridgwell, A., 2013. Marine ecosystem responses to Cenozoic global change, Science, 341(6145), 492-498.

Palmer, M. W. \& White, P. S., 1994. Scale Dependence and the Species-Area Relationship, The American Naturalist, 144(5), 717-740.

Polato, N. R., Gill, B. A., Shah, A. A., Gray, M. M., Casner, K. L., Barthelet, A., Messer, P. W., Simmons, M. P., Guayasamin, J. M., Encalada, A. C., Kondratieff, B. C., Flecker, A. S., Thomas, 
S. A., Ghalambor, C. K., Poff, N. L., Funk, W. C., \& Zamudio, K. R., 2018. Narrow thermal tolerance and low dispersal drive higher speciation in tropical mountains, PNAS, 115(49), 12471-12476.

Rahbek, C. \& Graves, G. R., 2001. Multiscale assessment of patterns of avian species richness, PNAS, 98(8), 4534-4539.

Rahbek, C., Borregaard, M. K., Colwell, R. K., Dalsgaard, B., Holt, B. G., Morueta-Holme, N., Nogues-Bravo, D., Whittaker, R. J., \& Fjeldså, J., 2019. Humboldt's enigma: What causes global patterns of mountain biodiversity?, Science, 365(6458), 1108-1113.

Roberts, G. G., White, N., \& Lodhia, B. H., 2019. The Generation and Scaling of Longitudinal River Profiles, Journal of Geophysical Research: Earth Surface, 124, 17.

Rolland, J., Condamine, F. L., Beeravolu, C. R., Jiguet, F., \& Morlon, H., 2015. Dispersal is a major driver of the latitudinal diversity gradient of Carnivora, Global Ecology and Biogeography, 24(9), 1059-1071.

Saupe, E. E., 2021. Explanations for tropical diversity gradients are rooted in the deep past, PNAS, 118(43), 1-3.

Schneider, U., Becker, A., Finger, P., Meyer-Christoffer, A., Ziese, M., \& Rudolf, B., 2014. GPCC's new land surface precipitation climatology based on quality-controlled in situ data and its role in quantifying the global water cycle, Theoretical and Applied Climatology, 115(1-2), 15-40.

Song, H., Huang, S., Jia, E., Dai, X., Wignall, P. B., \& Dunhill, A. M., 2020. Flat latitudinal diversity gradient caused by the Permian-Triassic mass extinction, Proceedings of the National Academy of Sciences of the United States of America, 117(30), 17578-17583.

Torrence, C. \& Compo, G. P., 1998. A practical guide to wavelet analysis, Bulletin of the American Meteorological Society, $\mathbf{7 9}(1), 61-78$.

Wapenhans, I., Fernandes, V. M., O’Malley, C., White, N., \& Roberts, G. G., 2021. Scale-Dependent Contributors to River Profile Geometry, Journal of Geophysical Research: Earth Surface, 126, 1-25.

Wessel, P., Luis, J., Uieda, L., Scharroo, R., Wobbe, F., Smith, W. H. F., \& Tian, D., 2019. The Generic Mapping Tools Version 6, Geochemistry, Geophysics, Geosystems, 20, 1-9.

Willig, M. R., Kaufman, D. M., \& Stevens, R. D., 2003. Latitudinal Gradients of Biodiversity: Pattern, Process, Scale, and Synthesis, Annual Review of Ecology, Evolution, and Systematics, 34, 273-309. 
a

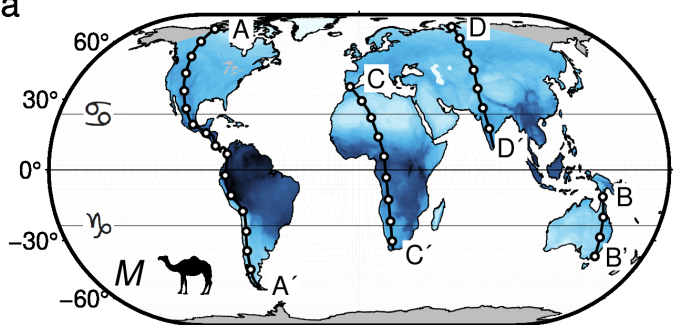

b

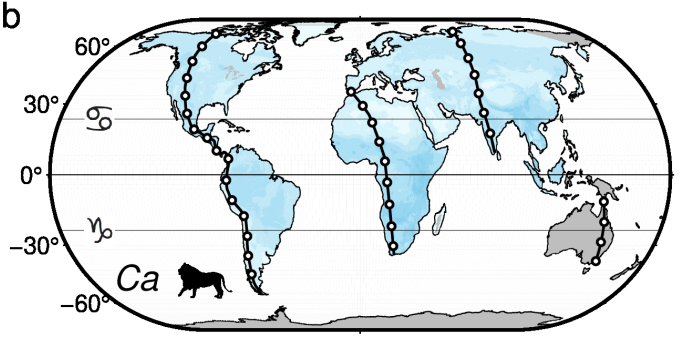

C

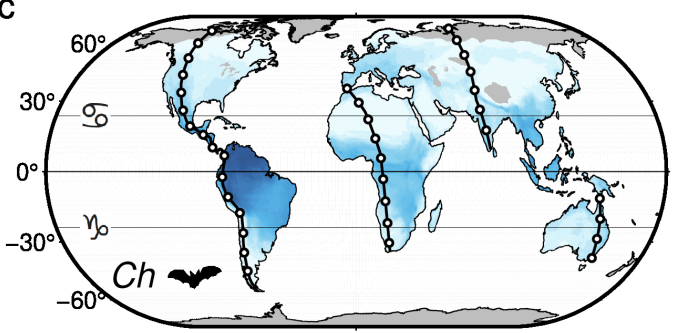

d

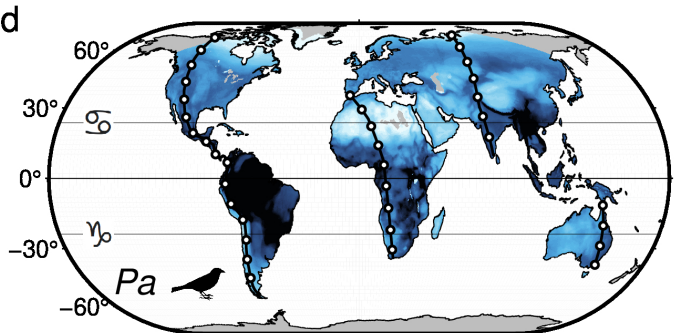

e

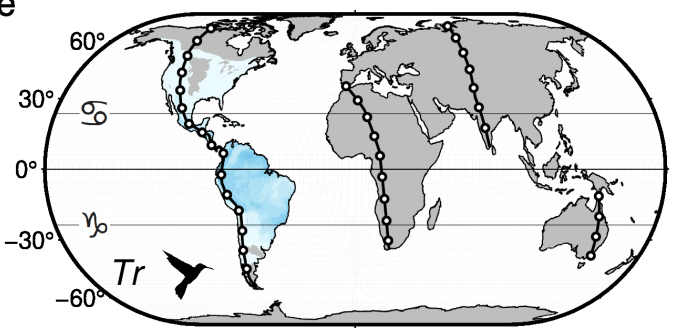

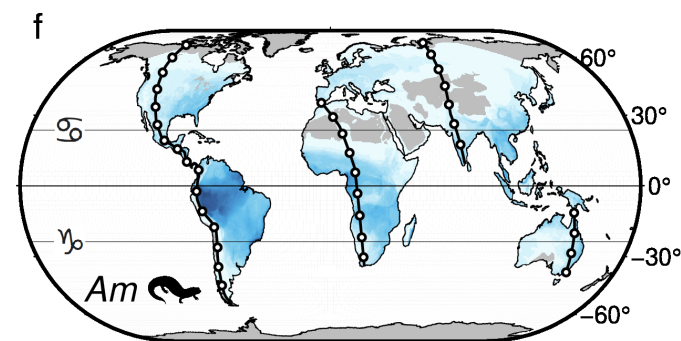

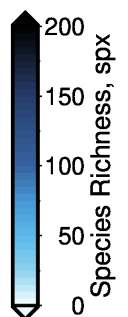
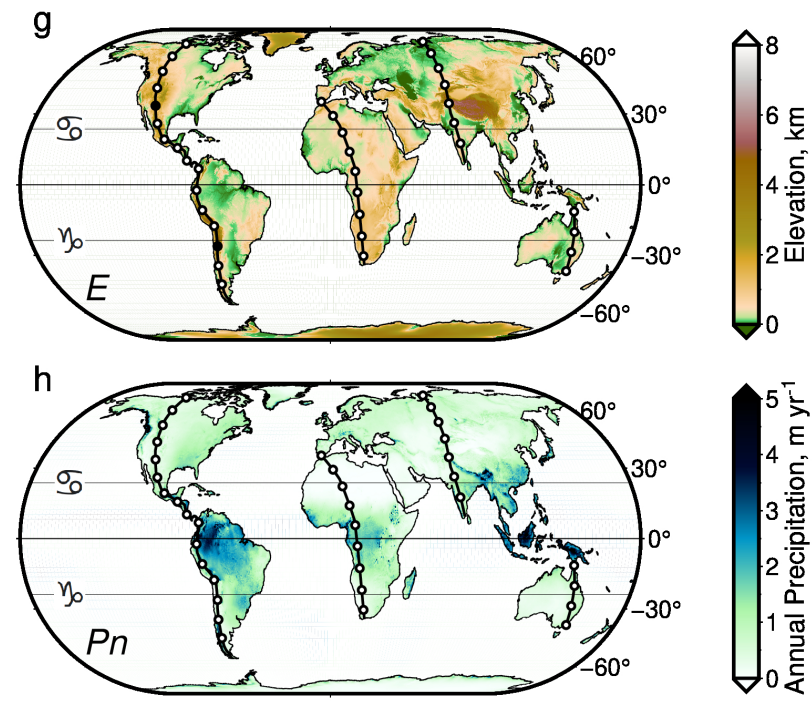

i
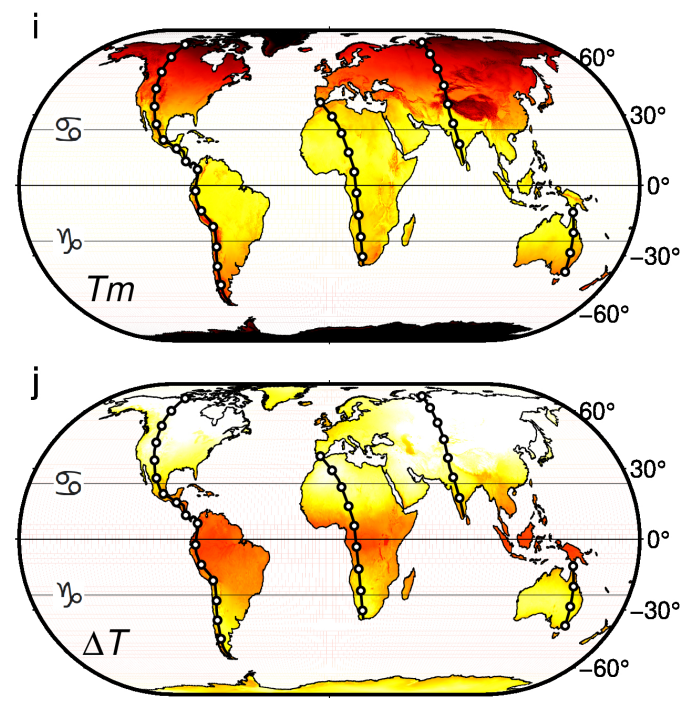

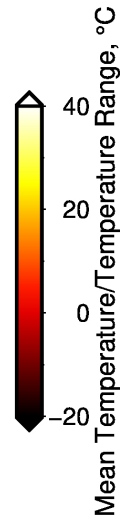

Figure 1: (a)-(f) Global patterns of species richness for all Mammalia $(M)$, Carnivora $(\mathrm{Ca})$, Chiroptera $(C h)$, Passeriformes $(P a)$, Trochilidae $(T r)$, Amphibia $(A m)$; spx $=$ species per $10 \times 10 \mathrm{~km}$ pixel (Jenkins et al., 2013); horizontal lines = Tropics of Cancer (northern), Capricorn (southern), and Equator; $\mathrm{A}-\mathrm{A}^{\prime}=$ transect through Americas investigated here; $\mathrm{B}-\mathrm{B}^{\prime}, \mathrm{C}-\mathrm{C}^{\prime}, \mathrm{D}-\mathrm{D}^{\prime}=$ transects investigated in Supporting Information. (g) Elevation $(E)$ from ETOPO1 global model with horizontal resolution of 1 arc-minute (Amante \& Eakins, 2009); filled circles on $\mathrm{A}-\mathrm{A}^{\prime}=$ Colorado Plateau/Mexican Highlands and Andean Altiplano. (h)-(j) Mean annual precipitation rate $(P n)$, temperature $(T m)$, and temperature range $(\Delta T)$ from 1981-2010 (Karger et al., 2017). 

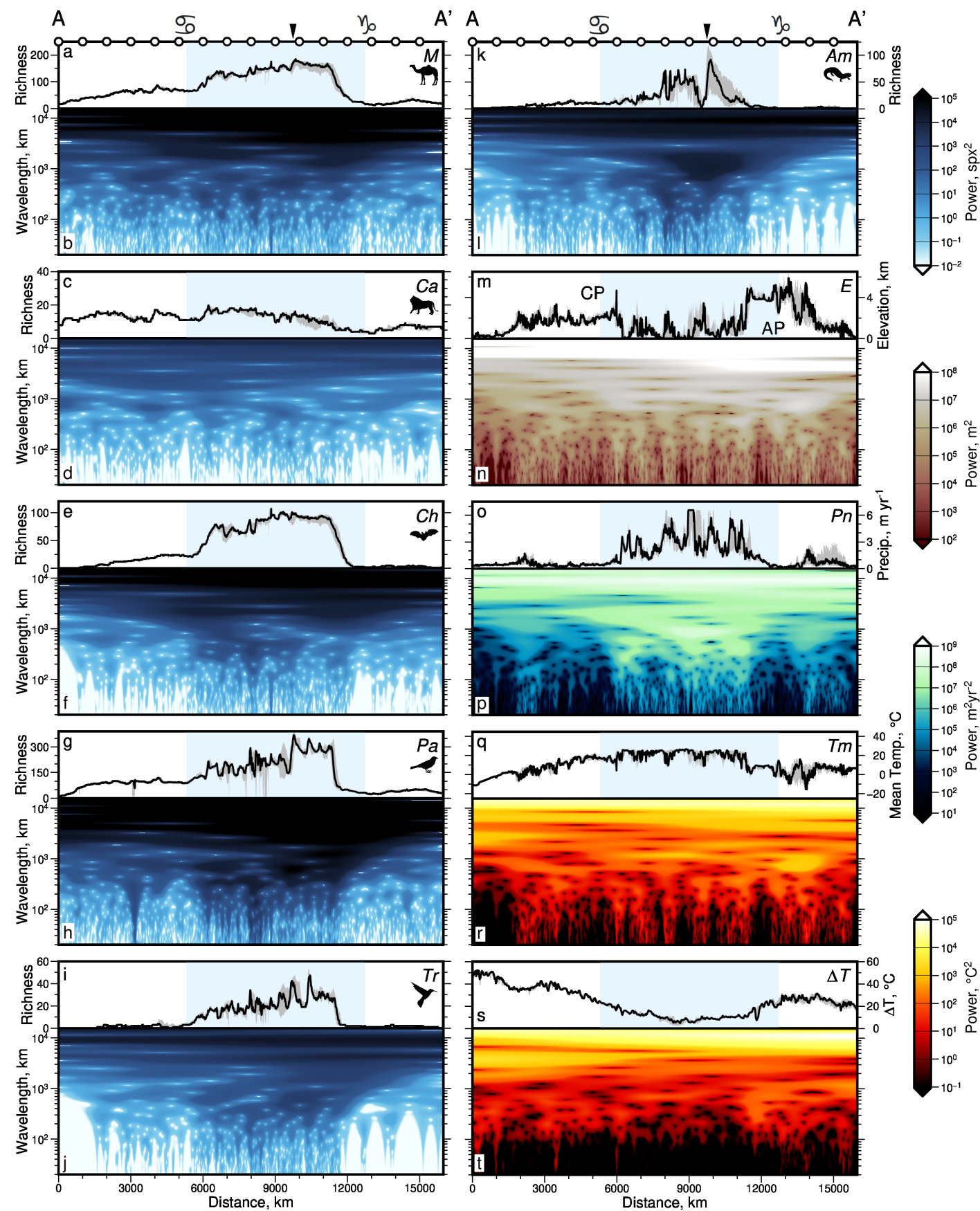

Figure 2: (a) Black line $=$ species richness of Mammalia $(M)$ along transect $\mathrm{A}-\mathrm{A}^{\prime}$; gray bands $=$ $100 \mathrm{~km}$ wide swaths centred on $\mathrm{A}-\mathrm{A}^{\prime}$; blue bands = tropical latitudes; white circles are shown every $1000 \mathrm{~km}$, see transect $\mathrm{A}-\mathrm{A}^{\prime}$ in Figure 1; black arrow and symbols above top axis = Equator and tropics as in Figure 1. (b) Continuous wavelet transform of Mammalia spatial series (black line in panel a). Colors $=$ spectral power as a function of location and scale (wavelength); $\mathrm{spx}=$ species per pixel. (c)-(t) As (a)-(b) but for Carnivora ( $\mathrm{Ca}$ ), Chiroptera ( $\mathrm{Ch})$, Passeriformes $(\mathrm{Pa})$, Trochilidae $(T r)$, Amphibia $(A m)$, elevation $(E)$, mean annual precipitation rate $(P n)$, temperature $(T m)$ and temperature range $(\Delta T)$ along transect $\mathrm{A}-\mathrm{A}^{\prime}$ (Amante \& Eakins, 2009; Jenkins et al., 2013; Karger et al., 2017). See Supporting Information for results for transects B- $\mathrm{B}^{\prime}, \mathrm{C}-\mathrm{C}^{\prime}, \mathrm{D}-\mathrm{D}^{\prime}$ and average global latitudinal transect. 

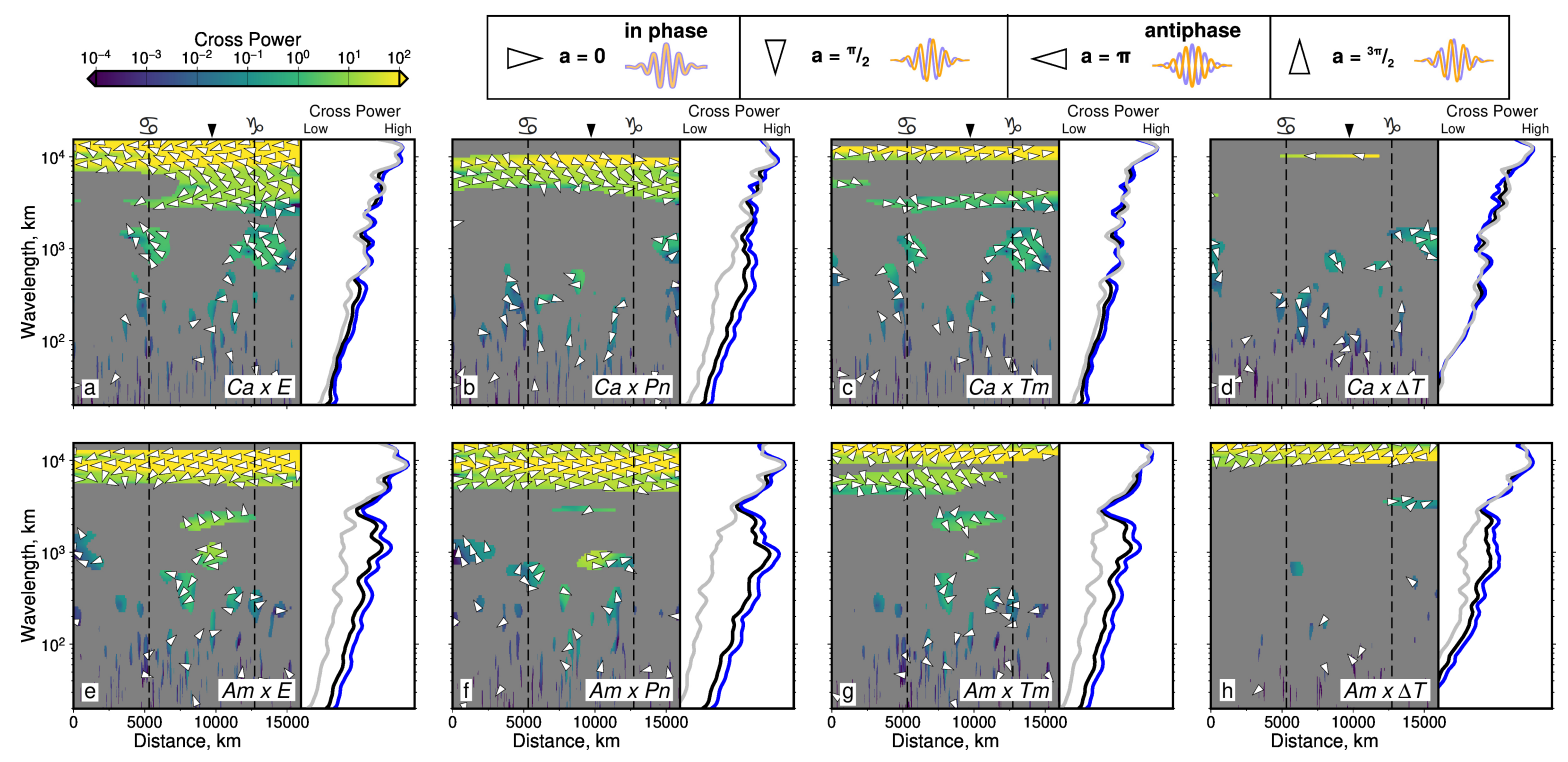

Figure 3: (a) Comparison of Carnivora $(C a)$ and elevation $(E)$ as a function of location and scale along transect $\mathrm{A}-\mathrm{A}^{\prime}$ (Figures 1-2). Colors = cross wavelet power; yellow = co-located large (positive or negative) amplitude signals. Gray masks regions with coherence below $90 \%$ significance level (see body text, Materials and Methods). Arrows = phase difference between spatial series: right/left pointing $=$ in-phase/anti-phase (see guide above panels b-d). Black arrow and symbols above plot $=$ Equator and tropics, as in Figure 1. Side panel: black/blue/gray lines $=$ distance-averaged cross wavelet power of all/tropical/non-tropical latitudes (see Figure 2). High cross power = large co-located amplitudes in the two spatial series. (b)-(d) Comparison of Carnivora and mean annual precipitation rate $(P n)$, temperature $(T m)$ and annual temperature range $(\Delta T)$. (e)-(h) Comparison of amphibian species richness and same environmental variables as panels $\mathrm{a}-\mathrm{d}$. 